\title{
Replica Plating and Rapid Ascus Collection of Neurospora
}

\author{
BY BARBARA D. MALING \\ Department of Biological Sciences, Stanford University, \\ Stanford, California, U.S.A.
}

(Received 19 March 1960)

\section{SUMMARY}

Two colonial strains of Neurospora crassa have been found suitable for testing nutritional requirements by replica plating. One strain (crisp) has been replicated with a set of needles and the other (crisp, ragged) with filter paper. When ripe perithecia from crisp $\times$ crisp crosses are submerged in water, complete asci can be collected rapidly with a capillary pipette.

\section{INTRODUCTION}

The methods commonly used for collecting asci and determining nutritional requirements have limited the usefulness of Neurospora crassa for genetic investigations which require large numbers of complete asci. Neurospora asci have usually been separated from the contents of a perithecium that had been squeezed open on the surface of a block of agar. The asci often adhere to one another at their bases and they may be hard to separate. The growth of strains with wild-type morphology is so rapid that simultaneous determination of the nutritional requirements of many isolates by replica plating is not possible. The nutritional requirements of the progeny of such strains must be determined by transferring each isolate to test tubes containing appropriate media. Some improved methods have been reported in recent years. Strickland (1960) developed a rapid method for collecting large numbers of unordered asci which is based on the observation that individual asci are shot forcibly from the perithecia. Many of these asci can be recovered from an apposing surface as a cluster of eight spores. Reissig (private communication) applied a variation of the replica plating technique to Neurospora. Unfortunately, his method cannot be used to determine nutritional requirements. This paper is a description of methods for replication of two colonial strains of Neurospora and for collection of asci from homozygous crosses of one of these strains.

\section{RESULTS}

Neurospora strains with mutations crisp and ragged. When the two morphological mutations $\operatorname{crisp}(\mathrm{cr}, \mathrm{B} 123)$ and ragged (rg, B53) (Perkins, 1959) are present in the same strain, growth is severely restricted. The colony formed has a diameter of only 2-3 mm. after 4 days' growth at $34^{\circ}$. When a dilute suspension of crisp, ragged conidia was spread on the surface of the agar in a Petri dish, the resulting colonies could be replicated by means of a continuous surface, such as velveteen or filter paper. The details of the replication procedure were essentially as described by Lederberg \& Lederberg (1952). The replica plates, incubated at $34^{\circ}$, could be scored 
3 days after replication ( $\mathrm{Pl}$. 1, fig. 1). Replication of crisp, ragged colonies arising from ultraviolet irradiated macroconidia was used to screen for nutritional mutants. A variety of nutritional, pigmented, and morphological differences can be scored in crisp, ragged strains. Crosses homozygous for both crisp and ragged produced very few ascospores and could not be used for recombination analysis.

Neurospora strain with crisp mutation. The single mutant strain crisp, B123 (an allele of crisp with more restricted growth than the standard allele isolated by Lindegren), was also suitable for replication and crosses of $\operatorname{crisp} \times$ crisp were fertile. Strains of $c$ risp produce profusely conidiating colonies about $5 \mathrm{~mm}$. in diameter after 2 days' growth at $\mathbf{3 4}{ }^{\circ}$. Colonies of crisp were replicated with needles so that the area of inoculation on the replica plates was small. This method of replication is similar to that developed by Forbes for Aspergillus nidulans (see Roberts, 1959). Asci are not shot forcibly from the perithecia of homozygous crisp crosses and they cannot, therefore, be collected by Strickland's method. However, large numbers of ordered asci were collected rapidly with the collection method (below) devised for these crosses. Moreover, ripe asci remained intact for a long time and they could be collected even after 3 months from cross-tubes which had been stored in the cold.

Collection of asci from homozygous crisp crosses. Asci could be collected beginning 3-4 weeks after inoculating the two parents together on to nutrient agar slopes. The top layer of agar, which contained embedded perithecia, was removed from the cross-tube and submerged in 10-15 ml. of sterile water in a Petri dish. When the cross was ripe, complete asci were extruded from the perithecia and settled to the bottom of the dish. The number of asci that were extruded could be increased by squeezing the perithecia. The asci were picked from the bottom of the dish and transferred to a block of $4 \%(\mathrm{w} / \mathrm{v})$ agar by means of a fine capillary pipette, suction being applied to the pipette by mouth through a rubber tube. The inside aperture of the capillary should not be much greater than the girth of an ascus. For greater efficiency, many asci are sucked up and blown out on to the centre of the agar block in one operation; these asci can then be picked up with the pipette, using additional water when necessary, and arranged so that the spores can be separated easily. The asci were routinely aged for 3-4 days at room temperature on the blocks to assure maximum germination; ageing may not be necessary when the crosses are older. When the spores were to be aged, the blocks were wetted with $5 \%(\mathrm{w} / \mathrm{v})$ sodium hypochlorite solution at least $10 \mathrm{~min}$. before use.

The ascospores may be separated at any time during the ageing period. Separated spores were transferred to supplemented medium and heat-shocked immediately. In our experiments the spores were germinated on Petri plates so that they could be tested for nutrient requirements by replication without further preliminary manipulation.

Replication of crisp. The replicator that was used consisted of 32 nichrome needles mounted on a rectangular brass plate; two long screws acted as stops for orienting the plate over the needles (Pl. 1, fig. 2). The needles were sterilized with a Bunsen burner. Proper positioning of ascospores on the master plate was facilitated by using the heated needles of the replicator to sear visible depressions in the agar before the spores were transferred to the plate. Inoculated plates were then heatshocked (with lids off) in a $60^{\circ}$ oven for $1 \mathrm{hr}$. When much less than $25 \mathrm{ml}$. of $2.5 \%(w / v)$ agar is used in the master plates, desiccation during heat shock may 
cause shrinkage and cracking, and the symmetry required for replication will be destroyed.

The plates were replicated 2-3 days after heat shock by inverting first the master plate and then the new plates on to the sterile needles. When more than one replica was desired, reseeding the replicator with the master was advisable before inoculating each new plate; reflaming the needles between successive replications from the same master plate was not necessary. The different types of testing media used in the replica plates were identified by adding Schilling food colouring to the medium. Contamination of the replica plates was negligible and crisp itself was never seen as a contaminant. All plates were incubated upside down to minimize contamination and condensation. The replica plates could be scored after 2 days' incubation at 34. ${ }^{\circ}$ (Pl. 1, fig. 3).

This work was part of a programme supported by research grants from the National Institute of Allergy and Infectious Diseases, U.S. Public Health Service (E 1462) and from the National Science Foundation (3840). The author was a predoctoral trainee in genetics under Public Health Service training grant 2G-158. The helpful suggestions of Drs D. D. Perkins, Patricia St Lawrence, W. N. Strickland and C. Yanofsky are gratefully acknowledged.

\section{REFERENCES}

Lederberg, J. \& Lederberg, E. M. (1952). Replica plating and indirect selection of bacterial mutants. J. Bact. 63, 399.

Perkins, D. D. (1959). New mutants and multiple point linkage data in Neurospora. Genetics, 44, 1185.

Roserts, C. (1959). A replica plating technique for the isolation of mutants, etc. J. gen. Microbiol. 20, 540.

Strickland, W. N. (1960). A rapid method for obtaining unordered Neurospora tetrads. J. gen. Microbiol. 22, 583. 


\section{EXPLANATION OF PLATE}

Fig. 1. Replication of crisp, ragged. The master plate is on the left and the replica on the right. (This plate was replicated with filter paper.)

Fig. 2. Replicator used with crisp (B 123).

Fig. 3. Replication of crisp. Each replica is labelled with the biochemical requirement it tests. The number below each colony on the master plate is the ascus and spore number $(1-1=$ ascus 1 , spore 1). The two visible characters (col \& al) do not show up clearly in the photograph. 

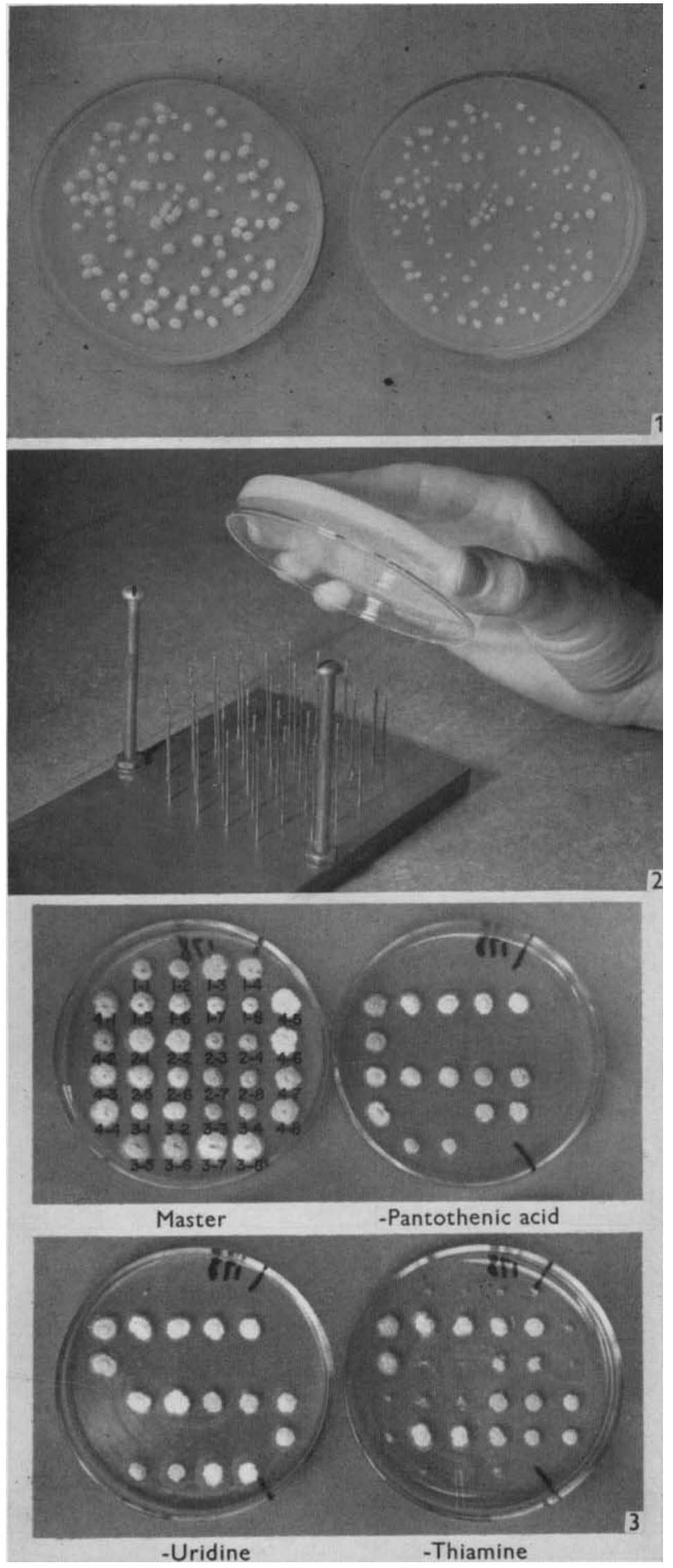

B. D. MALING

(Facing p. 260) 